PROCEEDINGS OF THE

AMERICAN MATHEMATICAL SOCIETY

Volume 130, Number 12, Pages 3453-3460

S 0002-9939(02)06479-1

Article electronically published on May 8, 2002

\title{
AFFINE HYPERSURFACES WITH GORENSTEIN SINGULAR LOCI
}

\author{
MONIQUE LEJEUNE-JALABERT AND RUTH I. MICHLER
}

(Communicated by Wolmer V. Vasconcelos)

\begin{abstract}
The main result of this paper presents necessary and sufficient criteria for the torsion module of differentials of an affine hypersurface with isolated singularities to be cyclic.
\end{abstract}

\section{INTRODUCTION}

The module of differentials of a nonsingular variety is torsion-free. Hence in order to study the torsion part of the module of differentials of a hypersurface with only isolated singularities one can confine oneself to a local study at the finitely many points in the singular locus of this variety. The module of differentials is defined using the universal Kähler differential. The dimension of the torsion part of the module of differentials is an important invariant of the hypersurface, the so-called Tjurina number.

In previous work the second author [3] found an ideal-theoretic description of the torsion part of the module of differentials that enables explicit computer algebra computations. In a subsequent paper [5], she proved that the torsion module of the module of differential one-forms of a singular plane curve is generated by at most two (and at least one) generator. In 6], she also exhibited examples of hypersurfaces $A$ with only isolated singularities in the affine space $\mathbb{A}_{K}^{N+1}$ over an algebraically closed field $K$ of characteristic zero that require an arbitrarily large number of generators for the torsion module of $\Omega_{A / K}^{N}$.

In this paper, we establish several necessary and sufficient criteria for the torsion module Torsion $\left(\Omega_{A / K}^{N}\right)$ of a hypersurface as above to be cyclic. We prove:

Theorem. Let $R=K\left[X_{1}, \ldots, X_{N+1}\right]$ be the polynomial ring in $N+1$ variables over an algebraically closed field $K$ of characteristic zero. Suppose that $F \in R$ defines a reduced hypersurface $A$ with isolated singularities in the affine space $\mathbb{A}_{K}^{N+1}$. Let $J=\left(\frac{\partial F}{\partial X_{1}}, \ldots, \frac{\partial F}{\partial X_{N+1}}\right)$ denote the Jacobian ideal, let $I:=(J, F)$ denote the ideal defining the singular locus $\operatorname{Sing}(A)$ in $\mathbb{A}_{K}^{N+1}$, and let $\left\{M_{i}\right\}_{i \in \Lambda}$ be the finitely

Received by the editors May 16, 2001 and, in revised form, July 2, 2001.

2000 Mathematics Subject Classification. Primary 13N05, 14J17, 14J70.

Key words and phrases. Quasi-homogeneous, differentials.

The first author was supported by C.N.R.S.

The second author was supported by NSF-DMS 0075057. She was killed in a tragic traffic accident in Boston on November 1, 2000. 
many maximal ideals in $R$ corresponding to points in Sing $(A)$. Then the following conditions are equivalent:

a) The torsion module Torsion $\left(\Omega_{A / K}^{N}\right)$ is a cyclic A-module.

b) The coordinate ring $T=R /(F, J)$ of $\operatorname{Sing}(A)$ is an Artinian Gorenstein ring.

c) $F \in J_{M_{i}}=J \otimes_{R} R_{M_{i}}$ for all $i \in \Lambda$.

d) $\operatorname{dim}_{K}\left[\operatorname{Torsion}\left(\Omega_{A / K}^{N}\right)\right]$ is maximal, i.e.

$$
\operatorname{dim}_{K}\left[\operatorname{Torsion}\left(\Omega_{A / K}^{N}\right)\right]=\sum_{i \in \Lambda} \mu_{i},
$$

where $\mu_{i}$ is the Milnor number of $A$ at $M_{i}$.

e) $F$ is locally analytically quasi-homogeneous in suitable analytic coordinates at each singular point $M_{i}, i \in \Lambda$.

The paper is organized as follows: We prove the equivalence of a) and b) in section 1. An alternative description of the torsion module of differentials and a reduction to the local case enable us to use duality for Artinian local rings. We prove the equivalence of $\mathrm{c}$ ) with $\mathrm{b}$ ), d) and e) in section 2 . We use results of E. Kunz [2], and B. Teissier [8] to prove b) $\Leftrightarrow$ c). The equivalence of c) with d) follows immediately from the equality of $\operatorname{dim}_{K}\left[\operatorname{Torsion}\left(\Omega_{A / K}^{N}\right)\right]$ with the Tjurina number. As for c) $\Leftrightarrow$ e), this is K. Saito's main Theorem in [7].

The last section contains explicit examples and historic remarks. In particular, for every integer $N \geq 1$, we construct an affine hypersurface $A=R /(F)$ in $\mathbb{A}_{K}^{N+1}$ whose torsion module Torsion $\left(\Omega_{A / K}^{N}\right)$ is cyclic, but such that no affine change of coordinates transforms its defining polynomial $F$ into a quasi-homogeneous polynomial.

\section{Hypersurfaces with Gorenstein singular loci}

In this section we prove the equivalence of assertions a) and b) of our Theorem. Let $K, R, F, J, I$ be as defined in the introduction. Throughout this paper we will use the following abbreviations:

$$
A:=R /(F), S:=R / J \text { and } T:=R / I .
$$

We consider the singular hypersurface

$$
X:=\operatorname{Spec}(R /(F))
$$

in the affine $(N+1)$-space which we assume to have only isolated singularities.

The only torsion modules of differentials are $\Omega_{A / K}^{N+1}$ and $\operatorname{Torsion}\left(\Omega_{A / K}^{N}\right)$, the torsion part of the module of $N$-forms. By Theorem 3 of [3], p. 82,

$$
\operatorname{dim}_{K}\left[\operatorname{Torsion}\left(\Omega_{A / K}^{N}\right)\right]=\operatorname{dim}_{K}\left(\Omega_{A / K}^{N+1}\right)=\operatorname{dim}_{K}(R / I)=: \tau(A)=\tau
$$

is finite. This number $\tau$ is called the Tjurina number of $A$.

In a previous paper 3 , the second author has given an ideal-theoretic description of the torsion module of differentials $N$-forms. She has proved:

\section{Proposition 1.1.}

$$
\operatorname{Torsion}\left(\Omega_{A / K}^{N}\right) \simeq(J: F) / J .
$$

We recall that the quotient ideal $(J: F)$ is defined as

$$
(J: F):=\{x \in R \mid x F \in J\} .
$$


Proposition 1.2. Let $F, R, J, I, S$ be as above. Then we have the following $S$ module isomorphim:

$$
\operatorname{Hom}_{S}(R / I, S) \simeq \frac{(J: F)}{J} .
$$

Proof. Consider the exact sequence

$$
0 \rightarrow I / J \rightarrow R / J \rightarrow R / I \rightarrow 0 .
$$

Applying the right exact functor

$$
\operatorname{Hom}_{S}(\cdot, S)
$$

to this short exact sequence, we obtain

$$
0 \rightarrow \operatorname{Hom}_{S}(R / I, S) \rightarrow \operatorname{Hom}_{S}(R / J, S) \stackrel{\rho}{\rightarrow} \operatorname{Hom}_{S}(I / J, S) .
$$

Recall that $R / J$ is abbreviated to $S$ and that

$$
\operatorname{Hom}_{S}(S, S) \simeq S,
$$

with the isomorphism given by $\theta \mapsto \theta(1)$. Note that for any $Q \in R$,

$$
\theta(Q \bmod J)=[Q \bmod J] \theta(1) .
$$

Therefore,

$$
\begin{aligned}
\operatorname{Ker}(\rho) & =\{\theta: R / J \mapsto S \mid \theta(I / J)=0\} \\
& =\{\theta \mid \theta(F \bmod J)=[F \bmod J] \theta(1)=0\} .
\end{aligned}
$$

By the exactness of the above sequence (1.2), it follows that $\operatorname{Hom}_{S}(R / I, S)$ is isomorphic to $(J: F) / J$ as an $S$-module.

Remark 1.3. Note that the identification of $\operatorname{Hom}_{S}(R / I, S)$ with $\operatorname{Torsion}\left(\Omega_{A / K}^{N}\right)$ given by Propositions 1.1 and 1.2 allows another proof of the duality Theorem 1.3 in [4] via the identification of $\Omega_{A / K}^{N+1}$ with $R / I$ via $d X_{1} \wedge \cdots \wedge d X_{N+1} \mapsto[1 \bmod I]$.

We now come to the reduction to the local case.

Definition 1.4. The singular locus of the hypersurface $A$ is defined by

$$
Z:=\operatorname{Sing}(A):=\operatorname{Spec}(R / I) \text {. }
$$

By assumption, the reduced singular locus $Z_{\text {red }}$ consists of the finitely many closed points

$$
Z_{\text {red }}:=\left\{o_{i}\right\}_{i \in \Lambda}
$$

where each closed point $o_{i}$ corresponds to the maximal ideal $M_{i} \subset R$ containing $I=(F, J)$ and $\Lambda$ is a finite indexing set. The maximal ideals $m_{i}=M_{i} /(F)$ of $A$, $i \in \Lambda$, are the elements of the support of the torsion module $\operatorname{Torsion}\left(\Omega_{A / K}^{N}\right)$, i.e.

$$
\operatorname{Supp}\left(\operatorname{Torsion}\left(\Omega_{A / K}^{N}\right)\right)=\left\{m_{i} \mid i \in \Lambda\right\} .
$$

Lemma 1.5 (cf. [5]). $\operatorname{Torsion}\left(\Omega_{A / K}^{N}\right) \cong \prod_{i \in \Lambda} \operatorname{Torsion}\left(\Omega_{A / K}^{N} \otimes A_{m_{i}}\right)$.

Lemma 1.6. Torsion $\left(\Omega_{A / K}^{N}\right)$ is a cyclic A-module if and only if $\operatorname{Torsion}\left(\Omega_{A / K}^{N} \otimes\right.$ $\left.A_{m_{i}}\right)$ is a cyclic $A_{m_{i}}$-module for each $i \in \Lambda$.

Proof. This follows immediately from Lemma 1.5 and the Chinese remainder theorem. 
Lemma 1.7 (cf. [4]). $S_{i}:=R_{M_{i}} / J_{M_{i}}$ is an Artinian local Gorenstein ring for each $i \in \Lambda$.

Proof. By Lemma 2.2 of [4], for all $i \in \Lambda$ the partial derivatives localized at $M_{i}$ form an $R_{M_{i}}$-sequence generating the localized Jacobian ideal $J_{M_{i}}$ of the regular local ring $R_{M_{i}}$. Now the assertion follows from Corollary 21.19 of [1], p. 537.

With the notation of Lemma 1.7 we recall

Definition 1.8. The number $\mu_{i}:=\operatorname{dim}_{K}\left(R_{M_{i}} / J_{M_{i}}\right)$ is called the Milnor number of the reduced hypersurface $A$ at $M_{i} \in \operatorname{Sing}(A)$.

Remark 1.9. In [3], p. 82 an example of a reduced plane curve $A$ with a single isolated singularity is given with $\operatorname{dim}_{K}(R / J)=\infty$. Therefore, in general Milnor numbers can only be defined locally.

Theorem 1.10. Suppose that $F \in R=K\left[X_{1}, \ldots, X_{N+1}\right]$ defines a reduced hypersurface ring $A=R /(F)$ with isolated singularities. Then its torsion module Torsion $\left(\Omega_{A / K}^{N}\right)$ is a cyclic A-module if and only if $T=R /(F, J)$ is an Artinian Gorenstein ring.

Proof. The support $\operatorname{Supp}\left(\operatorname{Torsion}\left(\Omega_{A / K}^{N}\right)\right)$ of the torsion module consists of the finitely many maximal ideals $m_{i}=M_{i} /(F)$ of $A, i \in \Lambda$, belonging to the singular locus $\operatorname{Sing}(A)$ of $A$. By Proposition 1.1, the canonical surjection

$$
A=R /(F) \longrightarrow T=R / I
$$

induces a $T$-module structure on $\operatorname{Torsion}\left(\Omega_{A / K}^{N}\right)$. By Lemma 1.6, $\operatorname{Torsion}\left(\Omega_{A / K}^{N}\right)$ is a cyclic $A$-module if and only if $\operatorname{Torsion}\left(\Omega_{A / K}^{N} \otimes A_{m_{i}}\right)$ is a cyclic $A_{m_{i}}$-module, hence equivalently a cyclic $T_{i}:=R_{M_{i}} / I_{M_{i}}$-module, for each $i \in \Lambda$. On the other hand, $T$ is an Artinian Gorenstein ring if and only if $T_{i}$ is an Artinian Gorenstein local ring, for each $i \in \Lambda$.

Thus we may assume that $R, A, S=R / J$ and $T=R / I$ are local rings. Furthermore, $T$ is Artinian. From the exact sequence

$$
0 \rightarrow I / J \rightarrow S \rightarrow T \rightarrow 0
$$

it follows that $T$ is a finitely generated $S$-module. Now Lemma 1.7 asserts that $S$ is a local Artinian Gorenstein ring. Let $\omega_{S}$ and $\omega_{T}$ be the canonical modules of $S$ and $T$, respectively. Then Proposition 21.4 of [1], p. 525 asserts that

$$
\begin{aligned}
\omega_{T} \cong \operatorname{Hom}_{S}\left(T, \omega_{S}\right) & \cong \operatorname{Hom}_{S}(T, S), \text { because } S \text { is Gorenstein, } \\
& \cong(J: F) / J \text { by Proposition } 1.2, \\
& \cong \operatorname{Torsion}\left(\Omega_{A / K}^{N}\right) \text { by Proposition } 1.1 .
\end{aligned}
$$

Now Proposition 21.5 of [1], p. 526 asserts that $T$ is Gorenstein if and only if $\omega_{T}$ is a cyclic $T$-module. By the above isomorphisms this is equivalent to $\operatorname{Torsion}\left(\Omega_{A / K}^{N}\right)$ being a cyclic $A$-module.

Remark 1.11. Theorem 3 of [3], p. 82 may be derived from duality for Artinian local rings as follows: From Proposition 1.1, Lemma 1.5 and what we have just proved above, we have an isomorphism

$$
\operatorname{Torsion}\left(\Omega_{A / K}^{N}\right) \cong \prod_{i \in \Lambda} \omega_{T_{i}}
$$


where $\omega_{T_{i}}$ is the canonical module of the local $\operatorname{ring} T_{i}$ of the coordinate $\operatorname{ring} T=$ $R /(F, J)$ of the singular locus of $A$ at $M_{i}$. Since $T_{i}$ is an Artinian local ring, $\omega_{T_{i}}$ is isomorphic to $\operatorname{Hom}_{K}\left(T_{i}, K\right)$ as a $K$-vector space by Proposition 21.4 of [1], p. 525 . Hence $\operatorname{dim}_{K} \omega_{T_{i}}=\operatorname{dim}_{K} T_{i}=: \tau_{i}$, and $\operatorname{dim}_{K}\left[\operatorname{Torsion}\left(\Omega_{A / K}^{N}\right)\right]=\sum_{i \in \Lambda} \tau_{i}=\tau$.

\section{Further CHARACTERIZATIONS}

Using Theorem 1.10 and important theorems due to E. Kunz 2], K. Saito [7, and B. Teissier [8] we show in this section that the torsion module Torsion $\left(\Omega_{A / K}^{N}\right)$ of a reduced hypersurface $A=R /(F)$ with isolated singularities is cyclic if and only if its defining polynomial

$$
F \in R=K\left[X_{1}, \ldots, X_{N+1}\right]
$$

is locally analytically quasi-homogeneous in suitable analytic coordinates at each singular point $M_{i} \in \operatorname{Sing}(A)$. Some other characterizations are given as well.

We keep the notation of the introduction and section 1 . The following proposition will be used in the proof of b) $\Leftrightarrow \mathrm{c}$ ).

Proposition 2.1. Suppose that $F \in R=K\left[X_{1}, \ldots, X_{N+1}\right]$ defines a reduced hypersurface ring $A=R /(F)$ with isolated singularities. Let $J$ be the Jacobian ideal and $I:=(F, J)$. For any $M_{i} \in \operatorname{Sing}(A)$, the following conditions are equivalent:

a) $I_{i}:=I \otimes R_{M_{i}}$ is generated by $N+1$ elements.

b) $F \in J_{i}:=J \otimes R_{M_{i}}$.

Proof. b) implies a) trivially. Assume a) holds. Since $R_{i}:=R_{M_{i}}$ is a local ring, Nakayama's lemma asserts that the $N+1$ generators of $I_{i}$ may be found among $F, \frac{\partial F}{\partial X_{1}}, \ldots, \frac{\partial F}{\partial X_{N+1}}$. Assume that $I_{i}=\left(F, \frac{\partial F}{\partial X_{1}}, \ldots, \frac{\partial F}{\partial X_{N}}\right) R_{i}$.

There is a surjection

$$
S_{i}=R_{i} / J_{i} \longrightarrow T_{i}=R_{i} / I_{i}
$$

Hence the Milnor number of $X=\operatorname{Spec} A$ at the point $M_{i}$, say $\mu_{i}(X)=\operatorname{dim}_{K} S_{i}$ is greater than or equal to its Tjurina number $\tau_{i}(X)=\operatorname{dim}_{K} T_{i}$. Now $\operatorname{dim}_{K} T_{i}$ is the intersection number of $X$ with the local polar curve

$$
\Gamma_{i}=\operatorname{Spec}\left(R_{i} /\left(\frac{\partial F}{\partial X_{1}}, \ldots, \frac{\partial F}{\partial X_{N}}\right)\right)
$$

at $M_{i}$. By Proposition 1.2 of B. Teissier [8, p. 317, this number is the sum of the Milnor number $\mu_{i}(X)$ and the Milnor number of the intersection $Y$ of $X$ with the hyperplane $X_{N+1}=0$ in $\mathbb{A}_{K}^{N+1}$ at $M_{i}$. Hence

$$
\mu_{i}(X) \geq \mu_{i}(X)+\mu_{i}(Y)
$$

Thus $\mu_{i}(Y)=0$ and $Y$ is smooth at $M_{i}$. Since multiplicity does not decrease by taking sections, $X$ is smooth at $M_{i}$, a contradiction. Hence $I_{i}=J_{i}$ and b) holds.

Proposition 2.2. Suppose that $F \in R=\left[X_{1}, \ldots, X_{N+1}\right]$ defines a reduced hypersurface $A$ with isolated singularities. Let $J$ be its Jacobian ideal and let $\left\{M_{i}\right\}_{i \in \Lambda}$, be the singular points of $A$. Then:

a) $\operatorname{dim}_{K}\left[\operatorname{Torsion}\left(\Omega_{A / K}^{N}\right)\right] \leq \sum_{i \in \Lambda} \mu_{i}$, where $\mu_{i}$ is the Milnor number of $A$ at $M_{i}$.

b) Equality holds in a) if and only if $F \in J_{i}:=J_{M_{i}}$ for all $i \in \Lambda$. 
Proof. Recall that

$$
\operatorname{dim}_{K}\left[\operatorname{Torsion}\left(\Omega_{A / K}^{N}\right)\right]=\sum_{i \in \Lambda} \tau_{i}
$$

where $\tau_{i}=\operatorname{dim}_{K}\left(R_{i} / I_{i}\right)$ is the Tjurina number of $A$ at $M_{i}$. Assertion a) follows immediately from the inequalities $\tau_{i} \leq \mu_{i}, i \in \Lambda$.

If equality holds in a), then $(*)$ implies that $\operatorname{dim}_{K}\left(R_{i} / I_{i}\right)=\operatorname{dim}_{K}\left(R_{i} / J_{i}\right)$ for all $i \in \Lambda$. Hence $I_{i}=J_{i}$, and $F \in J_{i}$ for all $i \in \Lambda$.

For the proof of the main result we recall the following definition from Kunz's article 2], p. 111.

Definition 2.3. Let $T$ be a Noetherian local ring, and let $\hat{T}$ be its completion. Then $\hat{T} \cong S / V$, where $S$ is a regular local ring, and $V$ is some ideal of $S$. Let $\mu(V)$ be the minimal number of generators of $V$. Then the ring $T$ is called an almost complete intersection, if

$$
d(T)=\mu(V)-(\operatorname{dim} S-\operatorname{dim} T)=1 .
$$

After all these preparations we can give the

Proof of the main theorem. Stated in the introduction:

a) and b) are equivalent by Theorem 1.10. Saito's main theorem of [7], p. 123, asserts that c) and e) are equivalent. Proposition 2.2 asserts that c) and d) are equivalent. Assertion c) implies b) by Lemma 1.7. It remains to show b) $\Rightarrow \mathrm{c}$ ).

Condition b) holds if and only if for every $M_{i} \in \operatorname{Sing} A$, the ring $T_{i}=R_{M_{i}} / I_{M_{i}}$ is Gorenstein. Thus, again we may assume that $R$, hence $T=R / I$ are local rings.

Since $\operatorname{dim} R=N+1$ and $\operatorname{dim} T=0$, the principal ideal theorem (Theorem 10.2 of [1], p.231) implies that the minimal number of generators $m$ of $I$ is bounded below by $N+1$; on the other hand, we have $m \leq N+2$. If $m=N+1, I$ is generated by an $R$-sequence of $N+1$ elements (see Corollary 17.7, p. 426 and Theorem 18.7, p. 451 of [1]); in other words, $T$ is a complete intersection, hence a Gorenstein local ring by Corollary 21.19 of [1], p. 537.

Therefore, in view of Proposition 2.1, the proof of b) $\Rightarrow$ c) will be completed, if we prove that $T$ may not be Gorenstein, if $m=N+2$, or in other words, if $T$ is an almost complete intersection. This is exactly what Corollary 1.2 of Kunz [2], p. 11 says.

To be precise, Kunz's Definition 2.3 assumes $R$ to be a complete local ring. To get our conclusion from his result, it is enough to observe the following two facts: Let $\hat{R}$ (resp. $\hat{T}$ ) be the completion of $R$ (resp. $T$ ) with respect to its maximal ideal. First $\hat{T}$ is Gorenstein if and only if $T$ is (Corollary 21.18 of [1, p. 537). Second $\hat{T}$ is a complete intersection (resp. an almost complete intersection) if and only if $T$ is. Indeed, since $R$ is a noetherian ring and $T$ is a finitely generated $R$-module, we have $\hat{T}=\hat{R} \otimes_{R} T=\hat{R} / I \hat{R}$ by Theorem 7.2 of [1, p. 181. Moreover, $\hat{R}$ is a flat $R$-module, hence we have $\hat{T}=\hat{R} / \hat{I}$ with $\hat{I}=I \hat{R}=I \otimes_{R} \hat{R}$. Now it follows from Nakayama's lemma that the minimal number of generators of $I$ (resp. $\hat{I}$ ) is $\operatorname{dim}_{K}(I / M I)\left(\right.$ resp. $\left.\operatorname{dim}_{K}(\hat{I} / \hat{M} \hat{I})\right)$ where $M($ resp. $\hat{M})$ is the maximal ideal of $R$ (resp. $\hat{R}$ ). Since $R / M=\hat{R} / \hat{M}=K$ and

$$
\hat{I} / \hat{M} \hat{I}=\hat{I} \otimes_{\hat{R}} \hat{R} / \hat{M}=\left(I \otimes_{R} \hat{R}\right) \otimes_{\hat{R}} \hat{R} / \hat{M}=I \otimes_{R} R / M=I / M I
$$

these numbers coincide. This completes the proof. 


\section{EXAMPLES AND REMARKS}

Our Theorem immediately implies that the torsion module Torsion $\left(\Omega_{A / K}^{1}\right)$ of a plane singular curve $A=R /(F)$ is cyclic, if $F$ is a quasi-homogeneous polynomial. But there are many other examples where condition c) of our main Theorem applies.

Example 3.1. Let $F=X^{7}+X^{6} Y^{3}+Y^{6} \in R=K[X, Y]$, where $K$ is an algebraically closed field of characteristic zero. Let $A=R /(F)$. Using identity $(*)$ below, it is easily checked that $M=(X, Y)$ is the unique maximal ideal of $R$ belonging to the singular locus $\operatorname{Sing}(A)$. We have

$$
\frac{\partial F}{\partial X}=7 X^{6}+6 X^{5} Y^{3} \text { and } \frac{\partial F}{\partial Y}=6 Y^{5}+3 X^{6} Y^{2}
$$

Hence

$$
\begin{aligned}
& F-\frac{1}{7} X \frac{\partial F}{\partial X}-\frac{1}{6} Y \frac{\partial F}{\partial Y}=-\frac{5}{14} X^{6} Y^{3}, \\
& 3 Y^{2} \frac{\partial F}{\partial X}-7 \frac{\partial F}{\partial Y}=Y^{5}\left(-42+18 X^{5}\right) .
\end{aligned}
$$

As $-42+18 X^{5} \notin M$, it follows that $Y^{5} \in J_{M}$, where $J$ denotes the Jacobian ideal of $A$. Therefore

$$
X^{6} Y^{2}=\frac{1}{3} \frac{\partial F}{\partial Y}-2 Y^{5} \in J_{M},
$$

and $F \in J_{M}$ by $(*)$. Thus $\operatorname{Torsion}\left(\Omega_{A / K}^{1}\right)$ is a cyclic $A$-module by the Theorem. One can check that the isomorphic $A$-module $(J: F) / J$ is generated by the residue class $\left[-7+3 X^{5}\right]$ modulo $J$.

Remark 3.2. Example 3.1 shows that condition c) of the Theorem does not imply that $F$ belongs to the Jacobian ideal $J$, in general. For if $F \in J$, then $\operatorname{Sing}(A)=$ $\operatorname{Spec}(R / I)=\operatorname{Spec}(R / J)$. However, $\operatorname{Spec}(R / J) \backslash \operatorname{Sing}(A)$ consists of 15 simple points as is easily checked. It follows that there is no affine change of coordinates which transforms $F$ into a quasi-homogeneous polynomial.

Remark 3.3. Theorem 2 of Zariski [9] p. 782 asserts that the length $l(T)$ of the torsion module $T$ of differentials of the coordinate ring $A=K\left[\left[X_{1}, X_{2}\right]\right] /(F)$ of an analytically irreducible algebroid curve is bounded by the length $c$ of the conductor $(\bar{A}: A)$ of $A$ in its integral closure $\bar{A}$ in the quotient field of $A$. He also proves that $l(T)=c$ if and only if in suitable coordinates $F=X_{2}^{n}-X_{1}^{m}$ with $(n, m)=1$ (Theorem 4 [9] p. 783). Our Proposition 2.2 and the main Theorem are affine analogues of these results. Moreover, our proof based on Proposition 1.1 extracted from [3], duality for Artinian local rings and Saito's main theorem [7, shows that, for any reduced algebroid curve, not necessarily analytically irreducible, the integer $l(T)=\operatorname{dim}_{K} T$ is bounded by the Milnor number, and equality holds if and only if $F$ is quasi-homogeneous in suitable analytic coordinates. Recall that if $F$ is analytically irreducible, $c$ coincides with the Milnor number.

For example, the torsion module of differentials is cyclic for all members of the family given by $F_{t}=X_{2}^{2}-X_{1}^{3}-t X_{1}^{2}=0$, because the dimension of the torsion module of differentials is equal to the Milnor number, namely 1 for $t \neq 0$ and 2 for $t=0$. 
Finally, we generalize Example 3.1 to show that for every integer $N \geq 1$ there is an affine hypersurface $A=R /(F)$ in $\mathbb{A}_{K}^{N+1}$ whose defining polynomial $F$ is not quasi-homogeneous, but the torsion module $\operatorname{Torsion}\left(\Omega_{A / K}^{N}\right)$ is cyclic.

Example 3.4. For any $N \geq 1$ the surface $A$ of $\mathbb{A}_{K}^{N+1}$ defined by

$$
F=X_{1}^{7}+X_{1}^{6} X_{2}^{3}+X_{2}^{6}+\sum_{i=3}^{N+1} X_{i}^{N+1} \in R=K\left[X_{1}, \ldots, X_{N+1}\right]
$$

satisfies condition c) of the Theorem. This is easily checked as in Example 3.1. Here again, $F$ does not belong to the Jacobian ideal, hence no affine change of coordinates transforms $F$ into a quasi-homogeneous polynomial.

\section{ACKNOWLEDGMENTS}

Most of this work was done when the second author visited the University of Versailles in July 2000 to collaborate with the first author, and she visited Northeastern University in Boston from August 1 until her death on November 1, 2000 under an NSF-POWRE grant, to collaborate with Professor Anthony Iarrobino and others. Both authors are indebted to him for drawing their attention to Kunz's paper [2], which helped to complete the proof of the main theorem.

\section{REFERENCES}

1. D. Eisenbud, Commutative algebra with a view toward algebraic geometry, GTM 150, Springer-Verlag, Heidelberg, (1994). MR 97a:13001

2. E. Kunz, Almost complete intersections are not Gorenstein rings, J. Algebra 28 (1974), 111115. MR 48:8496

3. R.I. Michler, Torsion of differentials of hypersurfaces with isolated singularities, J. Pure Appl. Alg. 104 (1995), 81-88. MR 96k:13036

4. R.I. Michler, The dual of the torsion module of differentials, Communications in Algebra (to appear).

5. R.I. Michler. On the number of generators of the torsion module of differentials, Proc. AMS 129 (2001), 639-646. MR 2001f:13041

6. R.I. Michler. Isolated hypersurface singularities with "large" Hochschild homology, AMS Contemporary Math. 266 (2000), 171-180. MR 2001k:13024

7. K. Saito, Quasihomogene isolierte Singularitäten von Hyperflächen, Inv. Math. 14 (1971), 123-142. MR 45:3767

8. B. Teissier, Cycles évanescents, sections planes et conditions de Whitney, Astérisque $\mathbf{7 / 8}$ (1973), 285-362. MR 51:10682

9. O. Zariski, Characterization of plane algebroid curves whose module of differentials has maximum torsion, Proc. Nat. Acad. Sci. USA 56 (1966), 781-786. MR 34:2576

Laboratoire de Mathématiques, UMR 8100 CNRS, 45 Avenue des Etats-Unis, UniverSité de Versailles-St. Quentin, F-78035, Versailles, France

E-mail address: lejeune@math.uvsq.fr

Department of Mathematics, University of North Texas, Denton, Texas 76203 